\title{
Diacronie
}

Studi di Storia Contemporanea

$N^{\circ} 7,3 \mid 2011$

«Spagna Anno Zero»: la guerra come soluzione

\section{Salazar et la Guerre civile espagnole}

\section{Cécile Gonçalves}

\section{(2) OpenEdition \\ Journals}

Édition électronique

URL : http://journals.openedition.org/diacronie/3255

DOI : 10.4000/diacronie.3255

ISSN : 2038-0925

\section{Éditeur}

Association culturelle Diacronie

\section{Référence électronique}

Cécile Gonçalves, «Salazar et la Guerre civile espagnole », Diacronie [En ligne], N 7, 3 | 2011,

document 8, mis en ligne le 29 juillet 2011, consulté le 01 mai 2019. URL : http://

journals.openedition.org/diacronie/3255; DOI : 10.4000/diacronie.3255 


\title{
Diacronie
}

8/

\section{Salazar et la Guerre civile espagnole}

\author{
Cécile GONCALVES *
}

La "Question espagnole" a déterminé la politique extérieure salazariste entre février 1936 et mars 1939. Par affinité idéologique et peur de la contamination rouge, Salazar (1889-1970) apporta son soutien à l'Espagne de Franco (1892-1975) pendant la guerre civile. Pourtant, l'alliance luso-britannique, que le leader de l'Estado Novo savait indispensable à la préservation de l'empire colonial portugais face à l'expansionnisme agressif des pays de l'Axe, n'allait point venir entraver ses efforts pour favoriser l'implantation en Espagne d'un régime autoritaire et nationaliste semblable au sien et qui ne constituerait plus une menace pour sa survie. 20000 portugais officiellement volontaires, les Viriatos, allaient combattre aux côtés des franquistes la République. Ainsi, après la victoire des nationalistes sur les forces loyales à la République, Salazar s'est exclamé depuis São Bento: "Nous avons gagné!».

\section{L'aide salazariste aux insurgés franquistes}

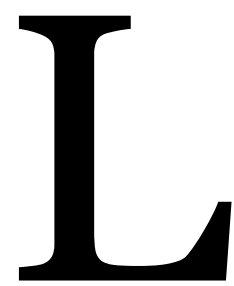

a victoire de la Frente Popular en février 1936 est venue interrompre un processus de normalisation des relations entre le régime dictatorial portugais et le gouvernement conservateur espagnol. L'amizade com a Espanha $^{1}$ signée entre le Portugal salazariste et le gouvernement espagnol de la CEDA devenait à présent caduc dans la mesure où une coalition de gauche ${ }^{2}$ se trouvait au pouvoir dans ce pays. Le régime salazariste ne pouvait que s’inquiéter de la montée des forces révolutionnaires en Espagne, Salazar et

\footnotetext{
1 Littéralement: "l'amitié avec l'Espagne”. Voir à ce sujet le livre de TORRE GOMEZ, Hipólito de la, Do "Perigo Espanhol" à amizade Peninsular. Portugal-Espanha 1919-1930, Lisboa, Editorial Estampa, 1985.

$2 \quad$ Entre avril 1931 et novembre 1933, l'Espagne républicaine a été gouvernée par une coalition de républicains radicaux et de gauche (avec les socialistes du PSOE), et son soutien aux émigrés politiques portugais provoqua un profond malaise dans les relations avec l'Estado
} 
l'Estado Novo n'ayant pas encore réussi à se remettre de l'expérience vermelha survenue en Espagne entre 1931 et fin 1933. Salazar, rappelons-le, s'est toujours considéré comme étant "en croisade" contre l'ennemi communiste. Issu d'une dictature militaire instaurée en 1926, l'Estado Novo, l' “Etat Nouveau” de Salazar ne peut, pour des raisons idéologiques, tolérer qu'un régime républicain, ayant pour fondement la souveraineté populaire, puisse cohabiter avec lui sur la même péninsule. Ses sympathies vont à l'Espagne nationaliste pour cette raison.

Dès que les résultats électoraux espagnols ont été connus, Salazar et les dirigeants politiques du régime portugais se sont alors inquiétés des conséquences fâcheuses qu'une telle victoire "rouge" allait irrémédiablement provoquer sur la stabilité et la sûreté de l'Estado Novo. En effet, la II République espagnole avait, entre 1933 et 1935, activement contribué à soutenir l'insurrection portugaise à l'encontre du régime de Salazar, notamment en offrant l'asile politique aux instigateurs des révoltes reviralhistas ${ }^{3}$. Peinant à mettre un terme aux attaques du Reviralho, le Gouvernement portugais redoute ainsi une possible contagion révolutionnaire. La victoire du Front populaire espagnol est vue comme une incitation à la révolte et les salazaristes craignent qu'elle ne ravive les aspirations des adversaires politiques que l'Estado Novo ne parvient pas à mater.

L'historien portugais César Oliveira (1941-1998) a bien décrit le mépris qu'éprouve le dictateur portugais à l'égard du gouvernement du Front populaire car celui-ci redevient à ses yeux, d'une manière encore plus radicale, le principal allié des nombreuses organisations et groupuscules d'opposition à l'Estado Novo portugais :

Tous les appuis apportés aux exilés portugais dans la lutte contre le régime dirigé par Oliveira Salazar entre avril 1931 et novembre 1933 ont été réactivé après les élections du 16 février 1936 qui donnèrent la victoire au Front populaire espagnol. Parmi ces appuis, on doit mettre en avant une station de radio, Rádio Fantasma, animée par des anarchistes en exil, la constitution d'une agence d'informations en Espagne dirigée par Novais Teixeira, des facilités pour l'impression et la diffusion du journal UNIR, organe du Front populaire portugais, qui, n'ayant pas obtenu un grand succès à l'intérieur du Portugal [mais] pris à Paris et en Espagne une certaine

Novo. Sur les relations luso-espagnoles entre 1931 et 1935, voir OLIVEIRA, César, Salazar e a Guerra Civil de Espanha, Lisboa, O Jornal, 1987.

3 Reviralhismo : terme donné à l'activité insurrectionnelle de l'opposition républicaine, démocrate et libérale entre 1926 et 1940 contre le régime dictatorial né du coup d'Etat militaire du 28 mai 1926. Voir à ce propos l'ouvrage de CARMO FARINHA, Luís Manuel do, $O$ Reviralho : revoltas republicanas contra a ditadura e o Estado Novo (1926-1940), Lisboa, Ed. Estampa, 1998. 
dimension et organisation lui permettant d'introduire beaucoup de propagande au Portugal à travers des réseaux ferroviaires espagnol et portugais4.

Salazar n'est pas directement intervenu dans les affaires espagnoles pour tenter de renverser la République. Cependant, depuis 1931, Lisbonne était devenue le centre de toutes les conspirations contre le régime républicain nouvellement installé à Madrid sous l'œil bienveillant du président du Conseil portugais. C'est du Portugal que le général Sanjurjo (1872-1936) a préparé l’insurrection militaire5 :

Le général Sanjurjo dirigea en août 1932 un coup d'Etat militaire de nature monarchique contre la République, en conséquence de quoi il fut fait prisonnier. Sous la législature d'un gouvernement CEDA/Parti Radical, dont le ministre de la défense était José Maria Gil Roblès, le général fut libéré [et s'exila au Portugal] dans l'Estoril courant 1934. Il était, en fait, le chef du soulèvement militaire de juillet 1936 et se trouvait au cœur de la conspiration, en étroite collaboration avec Mola, à l'intérieur de l'Espagne. L'avion piloté par Juan Ansaldo, qui, après le soulèvement, devait le ramener en Espagne, s'est écrasé pour excès de poids à la tentative de décollage d'un camp improvisé dans le maquis de Marinha, à Cascais, et Sanjurjo périt dans l'accident ${ }^{6}$.

L'ambassadeur de la République dans la capitale portugaise, l'éminent historien et ancien ministre des Affaires étrangères, Claudio Sanchez-Albornoz (1893-1984), abandonné par son personnel, est très vite traité comme un prisonnier dans sa propre ambassade. Les autorités portugaises tendent à considérer de plus en plus les insurgés de la cause nationaliste comme les représentants légitimes de l’Espagne. L'attitude de Pedro Teotónio Pereira (1902-1972), sous-secrétaire d'Etat puis ministre du Commerce et de l'Industrie, ne laisse pas de doutes quant aux penchants du gouvernement salazariste. Celui-ci dans une lettre adressée au président du Conseil portugais datée du 26 juillet 1936, propose de reconnaître officiellement l'Espagne "nationaliste” malgré la signature, somme toute peu contraignante, du traité de Non-intervention dans la guerre civile espagnole. Il écrit :

4 OLIVEIRA, César, Salazar e a Guerra Civil de Espanha, O Jornal, Lisbonne, 1987, p. 263.

5 Sur l'appui offert au "soulèvement" des généraux et de l'Armée espagnole par l'Estado Novo, voir DELGADO, Iva, Portugal e a Guerra Civil de Espanha, Lisboa, Europa América, 1982.

6 OLIVEIRA, César, op. cit., pp. 265-266. 
Le Portugal, sans attendre la décision collective des Puissances sur ce sujet, prend sur lui la responsabilité de reconnaître le gouvernement national espagnol. Les lois de bonne entente internationale nous obligent à expliquer les raisons de notre attitude et dire pourquoi nous l'avons fait sans l'accord préalable des autres puissances. La raison principale est que le pouvoir en place à Madrid n'appartient que nominalement au gouvernement légal qui s'avère être entre les mains du communisme qui prévoit dans son programme l'annexion du Portugal en vue de la constitution des soviets ibériques. Il gouverne, en effet, à Madrid une force politicomilitaire, dont la finalité immédiate est l'écrasement de l'armée nationale espagnole et la finalité seconde est une guerre révolutionnaire pour l'annexion du Portugal. Dès lors, le Portugal se considère en état de légitime défense, en état de danger imminent pour son indépendance. Il doit lui être reconnu, ainsi, le droit d'adopter, dans une urgence très grande, les mesures de défense nécessaires. Et la première est évidemment la rupture des relations diplomatiques avec ledit Gouvernement de Madrid, ce qui a pour avantage la fermeture du centre des conspirations qu'était à Lisbonne son ambassade; et qui entraîne, de manière logique, la reconnaissance du gouvernement national de Burgos, qui fait déjà régner sur la majeure partie de l'Espagne un ordre honnête et humain7.

Mais Salazar tient à se montrer prudent dans un premier temps afin de ne pas froisser sa vieille alliée. S’il n'a pas immédiatement suivi les conseils de son proche collaborateur, il rompt néanmoins les relations avec le gouvernement de Madrid le 23 octobre 1936. En décembre 1937, le Portugal envoie un représentant auprès du gouvernement de Burgos. Salazar s'est fait le porte-parole de la Junte de Burgos auprès de la Grande-Bretagne. Armindo Monteiro (1896-1955), ministre des Affaires Etrangères puis ambassadeur du Portugal à Londres, n'a cessé de vanter auprès des autorités britanniques les avantages qu'il peut y avoir pour l'Europe à soutenir la cause nationaliste espagnole contre l'expansion du communisme. Salazar, en agissant de la sorte, cherche à conquérir une plus grande autonomie vis-à-vis de l'alliance lusobritannique. Ainsi, il a longtemps joué sur les deux tableaux: il a, par intime conviction, prêté un appui sans équivoque au mouvement nationaliste tout en maintenant, simultanément, les apparences officielles auprès des autorités républicaines espagnoles comme le lui imposent à la fois les liens traditionnels avec l'alliée privilégiée, la Grande-Bretagne et le traité de Non-intervention dans la Guerre

$7 \quad$ Correspondência de Pedro Teotónio Pereira para Oliveira Salazar, (1931-1939), Lisboa, Comissão do Livro Negro sobre o Regime Fascista, 1987, vol.1, p. 48. Voir également TORRE GOMEZ, Hipólito de la, A Relação Peninsular na Antecâmara da Guerra Civil de Espanha (1931-36), Lisboa, Edições Cosmos, 1998. 
civile espagnole. Ainsi, Salazar ne reconnaîtra officiellement le gouvernement insurrectionnel de Franco qu'à compter du 28 avril 1938, soit, cinq mois après avoir nommé Pedro Teotónio Pereira comme ambassadeur du Portugal auprès de l'Espagne nationaliste et s'être accaparé le poste de ministre des Affaires Etrangères.

Lorsque éclate la Guerre civile espagnole, Oliveira Salazar sait que la consolidation et la pérennisation de son régime ainsi que la "conversion des âmes" qu'il a engagée vont dépendre de son dénouement. Si, au cours de cette Guerre civile, le sort des armes devait être favorable au camp républicain, les menaces à l'encontre de l'Estado Novo allaient vraisemblablement être multipliées. Le régime ne serait alors pas de taille à lutter et éprouverait toutes les difficultés du monde à y faire face. Il ne saurait résister, bien longtemps, aux actions déclenchées par les nombreuses oppositions jouissant de l'appui des partis espagnols qui constituaient le camp républicain. Le mouvement insurrectionnel du 18 juillet lui est apparu comme une opportunité à saisir, un fait providentiel qui va permettre l'implantation en Espagne d'un régime autoritaire et nationaliste comparable à l'Estado Novo et qui ne soit plus une menace pour la survie du régime portugais en s'offrant comme exemple à suivre.

Les Nationalistes espagnols, bien que moins religieux que le régime corporatif portugais, chérissent, il est vrai, quasiment le même idéal de société que le Doutor Salazar. Ce constat, sans compter les nombreuses facilités octroyées sur le territoire national aux Espagnols qui conspirent contre la République légale espagnole depuis 1931, légitime l'appui résolu, rapide et efficace que l'Estado Novo décide d'offrir à l'insurrection des militaires du 18 juillet 1936. Sebastião Ramirez (1898-1972), industriel originaire de l'Algarve et ancien ministre du Commerce, de surcroît grand ami de Gil Roblès (1898-1980), qu'il rencontre fréquemment à Madrid, coordonne toutes les actions et initiatives de soutien du régime portugais :

Oliveira Salazar, dès que l'insurrection militaire se déclencha, écrit César Oliveira, décida de soutenir avec prudence mais une grande détermination, le camp "national" contre la République. Sebastião Ramirez, industriel algarvien et ancien ministre du Commerce, coordonna les actions et initiatives de soutien. Celles-ci se focalisèrent sur les facilités de transports des hommes et du matériel de guerre à travers le territoire national, l'appui dans les stations de radio (surtout le Radio Club Português) et la presse, l'appui politique et diplomatique (surtout dans le Comité de Non-intervention en Espagne, siégeant à Londres), l'approvisionnement 
en denrées alimentaires, la viabilité de crédits bancaires et la concession de facilités pour recruter des soldats pour la Légion Etrangère au Portugal ${ }^{8}$.

Signataire d'un soit disant "pacte de non-intervention" avec Paris et Londres, Salazar ouvre pourtant les frontières portugaises aux rebelles nationalistes et laisse transiter par son territoire l'aide militaire accordée par Mussolini et Hitler aux troupes nationalistes de Franco. Nonobstant ce pacte de non-intervention et l'hostilité du Gouvernement britannique aux insurgés franquistes, aucun véritable contrôle n'a jamais pu être mis en place le long de la frontière luso-espagnole, laissant ainsi libre cours aux manœuvres salazaristes en faveur des insurgés franquistes.

Le président du Conseil portugais ne peut certes apporter à la rébellion qu'une aide militaire modeste. Le Gouvernement portugais a toutefois très ouvertement, autorisé le recrutement de volontaires pour l'Espagne ${ }^{9}$ : selon des estimations qui n'ont jamais été extrêmement précises - les engagés d'origine portugaise ne constituant pas une force militaire autonome - plus de 20.000 hommes, les Viriatos, ont été incorporés dans les armées franquistes. Si Salazar refuse de mettre à la disposition des insurgés nationalistes espagnols les aéroports militaires portugais, il fait comprendre à Quintanar que les autorités portugaises semblent ignorer tout de ce qu'il se passe dans les aéroports civils et particuliers. En d'autres termes, Salazar offre d'autres choses tout aussi précieuses que l'aide militaire octroyée par Mussolini ou Hitler : un lieu pour comploter, un refuge, et un moyen de communiquer d'une zone à l'autre au début de la guerre. Nicolas Franco, frère aîné du général, a ainsi pu installer son quartier général à Lisbonne pour les achats d'armes. La coopération entre Burgos et Lisbonne est donc totale. En conséquence, les républicains qui cherchent refuge en territoire portugais sont bien souvent remis aux Nationalistes - comme ce fut le cas pour Andrés de Castro, un avocat républicain, ainsi que vingt-quatre réfugiés de Vigo, qui ont été fusillés sur le pont international de Tuy. Dès le début, la presse portugaise est entièrement favorable aux Nationalistes. Le 20 août, le chargé d'affaires allemand à Lisbonne rend compte que le matériel de guerre transporté par les navires Wigbert et Kamerun ont été réexpédié sur l'Espagne sans problème, Salazar, dit-il, ayant levé « toutes les difficultés

8 OLIVEIRA, César «A Evolução Política » in SERRAO, Joel, OLIVEIRA MARQUES, António H. de (dir.), ROSAS, Fernando (coord.), Nova História de Portugal, vol.XII, Lisboa, Ed. Presença, 1992, p. 34. On consultera également avec profit l'article de GASPARD, Carlos, «Espanha, relações com a » in BARRETO, António e MONICA, Maria Filomena (coord.), Dicionário de História da Portugal, vol.7, suplemento A/E., Porto, Figueirinhas, 1999, pp. 648657.

9 ROSAS, Fernando, "Portugal e o Estado Novo (1930-1960) », in SERRAO, Joel, OLIVEIRA MARQUES, António H. de (dir.), op. cit., pp. 33-34. 
[...] grâce à son initiative personnelle et son habileté à régler tous les détails ».

\section{La conciliation de l'aide salazariste avec l'alliance luso- britannique}

Salazar a toujours fait preuve d'une grande prudence dans la concession de facilités aux insurgés franquistes. Il a veillé à ce que le soutien apporté à l'Espagne nationaliste se fasse dans le cadre des limites imposées par l'alliance luso-britannique. Le président du Conseil portugais ne s'est jamais véritablement engagé à fond, et il est passé maître dans l'art du compromis, dans la négociation des virages opportuns. Oliveira Salazar a mis un point d'honneur à garantir la compatibilité entre un traité « d'amitié et de bon voisinage avec l'Espagne », qui insiste particulièrement sur la sûreté des frontières communes, avec la fidélité à l'alliance entre le Portugal et l'Angleterre, même s’il dut préalablement contourner les pressions des autorités espagnoles "nationalistes" qui souhaitaient qu'un terme soit mis à cette alliance séculaire.

Il est certain que le gouvernement de Lisbonne saura profiter de la contrainte internationale de la Guerre civile espagnole pour gagner quelques espaces de manœuvre sur la scène internationale par rapport à la Grande-Bretagne, dans une certaine mesure pour redéfinir les termes de l'alliance, sans pour autant, s'éloigner d'elle sur l'essentiel. Faute de disposer d'une armée puissante, le chef de l'Estado Novo savait pertinemment que l'alliance s'avérait indispensable à la préservation de l'empire colonial portugais, dans une Europe en proie à l' "expansionnisme agressif" des régimes fasciste et national-socialiste ${ }^{10}$. L'appui aux militaires espagnols insurgés devait ainsi être octroyé sans que l'alliance britannique n'en souffre mais, parallèlement, celle-ci ne devait point constituer un frein aux efforts fournis par l'Estado Novo pour favoriser l'implantation en Espagne d'un régime qui ne soit ni une menace ni un exemple pour les forces de l'opposition.

Salazar a habillement su concilier et mener ces deux nécessités jusqu'à la fin de la guerre civile espagnole. 20 ooo portugais officiellement volontaires, les Viriatos, allaient ainsi combattre aux côtés des franquistes la République. La propagande profranquiste envahit la presse lusitanienne inféodée au régime et s'exerce massivement sur les Portugais. Dans le même temps, beaucoup de Républicains Espagnols qui espéraient trouver refuge au Portugal sont arrêtés et déportés par les autorités

$10 \quad$ L'Italie et l'Allemagne aspiraient à posséder ou à étendre leur présence coloniale. En ce sens, dans le courant des années 1930, la presse et les "coulisses diplomatiques" émettaient l'hypothèse de partager au profit de l'Allemagne certaines colonies portugaises. 
portugaises. Ce soutien, qui se veut discret, permettra notamment aux avions nazis de bombarder Guernica en avril 1937. Seule la "crise de Munich" a suscité quelques inquiétudes quant à cette position ambivalente du gouvernement portugais.

Les prétentions agressives du national-socialisme allemand sur la région des Sudètes et la Tchécoslovaquie, qui était liée par un traité d'amitié, de coopération et d'assistance à la France, ont rendu imminente la perspective d'une guerre en Europe centrale dès l'été 1938. Le problème pour le Portugal résidait alors dans le jeu des alliances conclu par le passé. En effet, si un conflit armé venait à éclater sur le vieux continent, les liens étroits unissant l'Espagne franquiste et l'Allemagne hitlérienne et l'alliance luso-britannique feraient que le Portugal de Salazar et l'Espagne de Franco se retrouveraient nécessairement dans des camps belligérants ennemis. Salazar, Pedro Teotónio Pereira, ambassadeur du Portugal auprès de l'Espagne "nationaliste", et plusieurs dirigeants politiques espagnols avaient conscience des périls qu'encourrait l' "amitié péninsulaire" dans l'éventualité d'un tel conflit. Comme l'a affirmé un dirigeant espagnol à P. Teotónio Pereira «il serait tragique pour les relations luso-espagnoles qu'éclate une guerre européenne sans que la nôtre soit liquidée ${ }^{11}$. Dès lors, en pleine "crise de Munich", Nicolas Franco, frère du Caudillo, et son représentant à Lisbonne, le marquis de Quintanar, ont cherché à s'entretenir avec Salazar, alors en vacances à Caramulo, courant septembre 1938, pour lui proposer un traité d'amitié et de sûreté des frontières. À la veille de la Seconde Guerre mondiale, la perspective d'un Traité luso-espagnol devenait de plus en plus effective. Ce traité sera d'ailleurs signé en mars 1939, quelques jours seulement après la fin de la Guerre Civile.

Ce traité stipulait qu'en cas de conflit armé européen, l'Espagne et le Portugal s'engageaient à ne pas s'envahir mutuellement. Après la signature du traité, Salazar a cherché à obtenir le consentement de la Grande-Bretagne. Armindo Monteiro ${ }^{12}$ a effectué les diligences nécessaires pour obtenir l'accord britannique. L’Angleterre - qui désirait garantir la neutralité de toute la péninsule Ibérique dans l'éventualité d'une guerre qui l'opposerait, conjointement à la France, à l'Allemagne hitlérienne - a considéré ce traité d' "amitié péninsulaire" comme une aubaine pour sa diplomatie. En effet, la signature par le Portugal, qui a toujours affirmé sa fidélité à l'alliance anglaise, d'un traité d'amitié avec l'Espagne nationaliste garantissait la possibilité d'utiliser le territoire portugais. Si considérable qu'ait pu être l'aide octroyée par Salazar, elle apparaît curieusement comme secondaire ; car la constitution d'un bloc ibérique n'a

11 Rapport secret de P. Teotónio Pereira, ambassadeur du Portugal à Madrid sur les antécédents du $1^{\text {er }}$ Protocole additionnel au Traité de 1939 entre Portugal et l'Espagne, Archives du MNE ; cité par OLIVEIRA, César, op. cit.

12 Lire à ce propos, le chapitre IV de l'étude de OLIVEIRA, César, op. cit. 
évidemment pas, sur le plan international, l'importance du rapprochement germanoitalien, conséquence directe de l'intervention des deux puissances dans la guerre civile espagnole. Devant la perspective d'une guerre européenne, les autorités britannique ont alors fermé les yeux sur l'aide apportée par Salazar au camp franquiste lors de la Guerre civile espagnole.

Bien avant la fin de la guerre civile espagnole, Salazar a attiré l'attention du gouvernement de Londres sur les dangers que représentaient pour les intérêts britanniques l'existence d'un régime franquiste allié à l'Allemagne hitlérienne et à l'Italie fasciste en cas de conflit européen. La préoccupation de préserver la sécurité de Gibraltar (et l'accès occidental à la Méditerranée), des côtes maritimes portugaises et de ses archipels atlantiques (points nodaux de la défense des routes atlantiques) amenèrent la Grande-Bretagne à se rapprocher des insurgés franquistes dès l'été 1937 et à considérer la position portugaise - qui incluait la neutralité en cas de conflit comme une condition indispensable au maintien de l'Espagne en dehors du conflit et pour la neutralisation globale de la péninsule Ibérique. Cela signifie que Londres a sciemment accepté de soutenir activement les efforts du Portugal neutre et de son ambassadeur à Madrid, Pedro Teotónio Pereira, auprès de Franco afin d'empêcher toute intervention espagnole dans le conflit. La publication récente de la correspondance privée entre l'ambassadeur Pedro Teotónio Pereira et Salazar ${ }^{13}$ vient confirmer, à ce propos, deux aspects essentiels : le rôle important - jusqu'à présent sous-évalué par une certaine historiographie étrangère - des pressions du gouvernement portugais pour contenir la faction germanophile et interventionniste du régime franquiste et le rôle du "Traité d'Amitié et de non-Agression luso-espagnol” du 17 mars 1939 et de son Protocole additionnel du 29 juillet 1940, comme instruments décisifs de la politique probritannique de neutralisation de la péninsule Ibérique.

\section{Soutenir les insurgés franquistes dans la Guerre civile d'Espagne pour renforcer son pouvoir au Portugal}

La Guerre civile espagnole a été l'occasion pour les autorités salazaristes de renforcer leur pouvoir sur l'ensemble de la société portugaise. En réalité, les menaces pesant sur la survie du régime en cas de victoire républicaine à l'issue de cette guerre et la nécessité d'apporter un large soutien au soulèvement des généraux, mené à partir d’octobre 1936 par le général Francisco Bahamonde Franco, ont été les principaux

13 Correspondência de Pedro Teotónio Pereira com Oliveira Salazar, voll.I-IV: (19391944), 1989-1991. 
facteurs qui ont permis une profonde modification dans les relations qu'entretenaient l'Estado Novo et la société portugaise :

Ce n'est qu'à partir du 1 octobre 1936 que le général Francisco Bahamonde Franco prit formellement la tête du camp nationaliste, du point de vue politique et militaire, conséquemment aux délibérations de la réunion de Salamanca tenue fin septembre et qui rassembla les principaux généraux et leaders des forces politiques soutenant le soulèvement. À la mort de Sanjurjo, le général Cabannelas avait pris la direction de la Junte gouvernementale, mais écarté de la réunion de Salamanca, il fut remplacé par Franco, dans les mains duquel se trouvaient concentrés la totalité du pouvoir de la zone nationale et le commandement suprême et effectif des troupes et milices ${ }^{14}$.

Avec la Guerre Civile d'Espagne, qui ravage le seul pays avec lequel le Portugal possède des frontières terrestres, on constate une accentuation des tendances fascistes du Estado Novo. En effet, le régime "s'endurcit", c'est-à-dire qu'il devient plus autoritaire et répressif, que ce soit dans le domaine de l'imposition coercitive de la volonté et des intérêts de l'Etat sur les citoyens, sur le plan de la concrétisation du contrôle de l'opinion publique, de la propagande et de l'encadrement politico-militaire des populations.

L'organisation de la Mocidade Portuguesa (MP) et la création de la Legião Portuguesa (LP) à l'été 1936; le renforcement du rôle des structures policières, notamment de la police politique (PVDE); la propagande autour du syllabaire nationaliste et du destin impérial du Portugal comme nation à vocation clairement coloniale et atlantiste, dynamisée et orchestrée par le Secrétariat de la Propagande Nationale (SPN); l'accélération de la mise en place des structures syndicales corporatives tout comme la politique extérieure portugaise (fonctionnant au service de la "cause nationale" incarnée par le général Francisco Franco) traduisent cet “endurcissement” et constituent les aspects concrets qui rapprochent l'Estado Novo salazariste des modèles fascistes. De plus, ce fut en avril 1936, peu de temps après la victoire du Front populaire en Espagne, qu'un décret-loi met en place la Colónia Penal - la colonie pénitentiaire - pour les prisonniers politiques et sociaux, à Tarrafal, île de Santiago dans l'archipel du Cap-Vert.

14 OLIVEIRA, César, « A Evolução Política » in op. cit., p. 34. 
Ce furent les Marins de l'Organisation Révolutionnaire Armée (ORA) qui, parmi d'autres détenus, inaugurèrent, en 1936, la Colónia Penal de Tarrafal. L'ORA était une structure subsidiaire du PCP et cherchait à organiser clandestinement les marins de la Marine de Guerre portugaise. En septembre 1936, l'ORA souleva des garnisons de navires de guerre qui ont tenté de sortir de la porte du Tejo pour aller appuyer la Marine de Guerre de la République espagnole, tentative infructueuse du fait des durs bombardements subis qui les empêchèrent de sortir de cet estuaire ${ }^{15}$.

Avec la Guerre Civile espagnole, le régime portugais commence à se soucier de l'encadrement des masses. Il développe une inquiétude profonde quant à l'organisation des masses populaires autour d'un holotype nationaliste. Cette préoccupation survenue dès 1936 et l'arrivée au pouvoir du Front républicain en Espagne fait que l'on peut légitimement affirmer, que l'Estado Novo acquiert une dimension mobilisatrice des masses, contrôlée par le gouvernement.

Oliveira Salazar, président du Conseil et ministre des Finances depuis 1932, renforce à partir de mai 1936, son pouvoir au sein de l'édifice politique et institutionnel de l'Estado Novo. En effet, en mai 1936, le leader du régime s'est emparé du poste de ministre de la Guerre (qu'il ne va abandonner qu'en septembre 1944) et, à partir de novembre de la même année, après avoir évincer Armindo Monteiro, il s'est rendu maître du ministère des Affaires Etrangères. Tout cela signifie qu'au-delà de l' "endurcissement" que connaît le régime dès la victoire du Front populaire en Espagne (février 1936) et surtout après la déflagration de la Guerre Civile, l'Estado Novo est progressivement devenu synonyme de dictature personnelle, de concentration des pouvoirs entre les mains de son chef : Salazar, bien que Premier ministre et chef du parti unique l'União Nacional, est également ministre des Finances, de la Guerre et des Affaires Etrangères. Il occupe et dirige, dans un contexte de crise européenne et d'impact de la Guerre Civile d'Espagne, les principaux ministères :

La crise européenne qui déflagra dans les années trente était due, principalement, à l'expansionnisme agressif du national-socialisme allemand, qui, à partir de janvier 1933, avec Hitler prendra le pouvoir en Allemagne, et à la crise de la Société des Nations (SDN), concrétisée par l'invasion de la Manchourie par le Japon, l'agression de l'Italie de Mussolini envers l'Ethiopie, membre fondateur de la SDN, et l'abandon officiel de la SDN par l'Allemagne en 1935. Les aspirations de l'Allemagne sur le Dantzig, les désirs d'annexion de l'Autriche (ce qui arrivera en

$15 \quad$ Ibidem, p. 35. On pourra également lire ROSAS, Fernando, "O Estado Novo (19261974) », in MATTOSO, José (dir.), História de Portugal, vol.VII, Lisboa, Círculo de Leitores, 1994. 
mars 1938) et la tentative de rassembler tous les peuples de langue allemande dans le III ${ }^{e}$ Reich marquèrent la crise européenne qui conduira à la guerre sous la complaisance des démocraties occidentales durant l'escalade agressive dirigée par Adolf Hitler ${ }^{16}$.

Les conséquences de la Guerre Civile espagnole ne se sont pas simplement fait ressentir au sein de l'establishment de la dictature salazariste. Les divers groupes de l'opposition à l'Estado Novo ne sont pas restés indifférents aux événements d'Espagne.

Dans les faits, les nombreuses forces d'opposition au régime institutionnalisé par Oliveira Salazar ont retrouvé, depuis février 1936, le souffle nécessaire pour relancer leurs tentatives d'insurrection, qui avaient été jusqu'alors mises en sommeil notamment après la répression sanglante du mouvement du 18 janvier 1934 et l'emprisonnement en 1935 des principaux dirigeants du parti communiste. Depuis la victoire du Front populaire aux élections de février 1936, il n'est pas rare d'entendre et de voir en Espagne, lors des manifestations de soutien aux républicains des discours, des "mots d'ordre", des pancartes ou, plus simplement, des distributions de pamphlets, contre le régime portugais :

L'hostilité de la part des partis composant le Front Populaire espagnol envers l'Estado Novo fut une constante, habilement instrumentalisée par la presse inféodée au régime portugais et au SPN pour manipuler l'opinion publique contre le péril de "l'union ibérique" que la propagande du régime portugais associait aux partis du Front populaire espagnol ${ }^{17}$.

Après la période que certains historiens ont qualifié de bieno negro, "les deux années noires”, (novembre 1933-février 1936), sous laquelle s'est opéré un rapprochement entre la République espagnole et l'Estado Novo (ce qui a permit la répression de la Révolte des Asturies, dans laquelle s'étaient enrôlés des émigrés politiques portugais), la victoire de la gauche espagnole aux élections de février a de nouveau fait renaître parmi les oppositions à l'Estado Novo l'espoir dans la possibilité d'entraver sa survie :

La répression de la Révolte des Asturies (septembre/octobre 1934) retombera sur de nombreux exilés portugais, qui avaient fourni les armes et munitions, qu'ils

16 OLIVEIRA, César, "A Evolução Política » in op. cit., p. 36. On pourra également consulter à ce propos, OLIVEIRA, César, Cem anos nas relações luso-espanholas, Lisboa, Ed. Cosmos, 1995.

17 OLIVEIRA, César, « A Evolução Política » in op. cit., pp. 36-37. 
avaient acquis pour la lutte contre l'Estado Novo aux socialistes et mineurs asturiens. Beaucoup d'entre eux furent faits prisonniers dès octobre 1934 jusqu'en février 1936 comme leaders des mineurs (ce fut le cas, parmi d'autres, du capitaine Alexandrino dos Santos et du lieutenant Oliveira Pio), d'autres réussir à fuir vers la France et la Belgique, retournant en Espagne après février $1936^{18}$.

Communistes, anarchistes et démocrates ont pu rétablir des liaisons avec les différents groupes et personnalités des oppositions en Espagne, ils y ont à nouveau trouvé des soutiens, qui avaient dû s'exiler en attendant le retour de la République.

La première grande action déclenchée sur le territoire portugais contre l'Estado Novo, après le début des hostilités en Espagne, a été l'insurrection des agents de la Marine le 8 septembre 1936, qui ont tenté de s'emparer de plusieurs navires de guerre et sortir par la porte du Tejo afin d'aller soutenir la République espagnole. Si le début de la Guerre Civile d'Espagne a créé des conditions propices au renforcement des capacités d'initiatives des oppositions au régime incarné par Salazar, l' "endurcissement" dudit régime a entraîné une violence jusque-là inédite et l'instauration de mesures policières que l'Estado Novo commence à appliquer dès juillet 1936.

Durant l'année 1937, des groupes anarchistes organisent une série d'actions contre le régime. De janvier à juillet, divers attentats à la bombe, dont le plus important reste l'attentat raté contre Salazar, ont été perpétrés à Lisbonne, visant différents objectifs directement liés au soutien apporté par le Portugal à la cause nationaliste du général Franco. Même si ces actions ont permis l'affirmation de la cohérence des anarchistes portugais, cette série d'attentats a été instrumentalisé par les services de propagande de l'Estado Novo comme ce fut le cas pour la répression de la révolte des marins. Le SPN cherche un moyen efficace auprès de la population portugaise pour discréditer la résistance portugaise en démontrant les liaisons existantes entre "ces éléments perturbateurs de l'ordre public» et les «rouges espagnols », afin de renforcer la cohésion des forces sociales et politiques qui les soutenaient. Les "rouges portugais" sont alors considérés comme des ennemis inassimilables, à la solde des républicains espagnols qui souhaitent ardemment la disparition de la Nation portugaise et oeuvrent à l'avènement d'un grand soviet ibérique :

18 Ibidem, p. 37. Voir également l'ouvrage de VINCENTE, António Pedro, Espanha $e$ Portugal. Um olhar sobre as relações Peninsulares no séc. $X X$, Lisboa, Tribuna da História, 2003. 
Lors du congrès de Saragosse de la CNT, en mai 1936, la délégation de la CGT portugaise, venue de la clandestinité et de l'intérieur du Portugal, s'est engagée à déclencher des actions contre l'Estado Novo au cas où les forces conservatrices concrétisent des actions contre la République. Les attentats perpétrés à cet effet par les anarchistes courant 1937 - Casa de Espanha, des dépôts de combustible de bétail, Rádio Club Português, ministère de l'Education et, finalement, l'attentat contre Salazar le 4 juillet, sur Avenidas Novas, quand il descendait de voiture pour assister à la messe - découlèrent de cet engagement et furent faits avec des explosifs apportés au Portugal par l'exilé en Espagne "Silva da Madeira". Les attentats ont été les dernières actions perpétrées par les anarchistes portugais. La détention de presque tous les impliqués, et pour de nombreuses années, décima définitivement les anarchistes portugais qui avaient encore des activistes après la grande répression de $1934^{19}$.

$\mathrm{Au}$ commencement du Estado Novo salazariste, les membres des anciens mouvements considérés comme les plus radicaux tels l'Intégralisme Lusitanien, le mouvement National-Syndicaliste de Rolão Preto (1893-1977), la Croisade Nationale Nuno Alvares Pereira et la Ligue du 28 mai, ont proposé la création d'une milice populaire paramilitaire, inspirée d'organisations comme les SA (Sections d'Assaut) nationales-socialistes ou la MVSN (chemises noires fascistes) italienne. Salazar s'était alors opposé à la mise en place d'une telle organisation car il n'éprouve pas le besoin d'appuyer son autorité sur une milice car contrairement à ce qui est advenu avec les nazis en Allemagne ou les fascistes en Italie, l'action des milices n'a pas été déterminante dans l'ascension vers le pouvoir.

Cependant, la nécessité de canaliser les aspirations de ces éléments radicaux mais aussi devant la menace que constitue pour le pays la Guerre Civile Espagnole et l'hostilité de certains mouvements politiques radicaux qui prétendent incorporer le Portugal dans une future Union Soviétique Ibérique rend l'idée acceptable aux yeux de Salazar. Sur une proposition du Major Jorge Botelho Moniz (1898-1961), le gouvernement de Salazar décide la création de la Legião Portuguesa. La LP ne serait pas une milice partisane comme le sont la SA ou la MVSN, mais serait un organisme d'Etat, dépendant directement des ministères de l'Intérieur et de la Guerre. Ainsi, par le Décret-Loi n. ${ }^{\circ} 27058$ du 30 septembre 1936 est institutionnalisée la Légion Portugaise avec l'objectif principal de « défendre le patrimoine spirituel de la Nation et combattre la menace communiste et l'anarchisme ».

19 SANTANA, Emídio, Historía de Um atentado, Lisboa, Publicações Fórum Lda., 1976, cité par OLIVEIRA, César, « A Evolução Política » in op. cit., p.37. 
La Mocidade Portuguesa, organisation mise en place la même année et visant à l'encadrement idéologique de la jeunesse, et la Legião se complètent. Toutes deux visent à la "conversion des âmes" des Portugais, elles sont des organisations au service de la défense des principes politiques du régime, la première prenant en charge la jeunesse et la seconde obéissant à un régime de volontariat et n'acceptant dans ses rangs que des hommes de plus de 18 ans. De ses rangs seront issus les futurs Viriatos qui allaient combattre aux côtés de forces franquistes dans la "Croisade contre le communisme”.

Lors du rassemblement anticommuniste au Campo Pequeno de Lisbonne le 28 août 1936, Botelho Moniz, devant des milliers de participants, parmi lesquels José Maria Péman (1897-1981), a affirmé :

La Guerre Sainte va commencer, la guerre de tous les instants. La croisade héroïque va commencer à laquelle nous convions tous les Portugais [...] Nous, les nationalistes, sommes légion et sommes portugais. Nous constituons la Legião Portuguesa, la légion où n'entrent que des vrais "Portugais", mais qui reste ouverte à tous les Portugais, loyaux, disciplinés, dignes et honorés, qui acceptent le slogan "Pour la Famille, pour la Patrie, pour la Civilisation Lusitanienne"20.

Salazar qui honnie plus que tout le communisme et se méfie des ambitions d'unification de la péninsule Ibérique du Front populaire décide d'apporter son soutien aux généraux insurgés du mouvement de Franco. Il ouvre alors les frontières portugaises aux rebelles et laisse transiter les armes fournies par l'Italie fasciste et l'Allemagne national-socialiste. La propagande pro-franquiste s'exerce activement sur les Portugais. Permettre à un régime nationaliste et autoritaire de s'implanter en Espagne s'était s'assurer de la pérennisation et la sûreté de son propre régime. La "Croisade" de Franco contre le Front populaire espagnol démocratiquement élu devient celle de Salazar car pour ce dernier, la péninsule Ibérique est une et indivisible en matière de défense. L'aide de Salazar dans les premiers mois du conflit s'avère absolument décisive dans la victoire des troupes de Franco en 1939. La Guerre Civile espagnole a permis la fascisation du régime portugais car la mobilisation des masses va devenir une préoccupation majeure du régime. Il s'agissait de faire de cette guerre "une guerre de civilisation” à laquelle tout le peuple devait se rallier. Sur le champ de bataille espagnole se jouait le destin de l'Europe, de l'Occident chrétien. Ainsi, après la victoire

\footnotetext{
20 Discours de José Maria Pémen du 28 août 1936 cité par VINCENTE, António Pedro, op. cit., p. 220.
} 
des nationalistes sur les troupes loyales à la République, Salazar s'est exclamé du balcon de São Bento: "Nous avons gagné!", c'est-à-dire que leur conception autoritaire du pouvoir a vaincu, par la force, l'hérésie démocratique. 


\section{* L'auteur}

Gonçalves Cécile a comme domaine de recherche l'histoire contemporaine du Portugal. Elle est : doctorante en Etudes politiques à l'Ecole des Hautes Etudes en Sciences Sociales, Paris ; rattachée au Centre d'Etudes Sociologiques et Politiques Raymond Aron (CESPRA) ; doctorante en Histoire contemporaine à l'Université Picardie Jules Vernes, Amiens ; et rattachée au Centre d'Histoire des Sociétés, des Sciences et des Conflits, Faculté d'histoire et de géographie.

URL: < http://studistorici.com/progett/autori/\#Gonçalves >

\section{Per citare questo articolo:}

GONÇALVES, Cécile, «Salazar et la Guerre civile espagnole», Diacronie. Studi di Storia Contemporanea: Spagna Anno Zero: la guerra come soluzione, 29/07/2011,

URL:< http://www.studistorici.com/2011/07/29/goncalves_numero_7>

Diacronie Studi di Storia Contemporanea $\beta$ www.diacronie.it

Risorsa digitale indipendente a carattere storiografico. Uscita trimestrale. redazione.diacronie@hotmail.it

Comitato di redazione: Marco Abram - Giampaolo Amodei - Jacopo Bassi - Luca Bufarale - Alessandro Cattunar - Alice De Rensis Barbara Galimberti - Deborah Paci - Fausto Pietrancosta - Martina Sanna - Matteo Tomasoni - Luca Zuccolo 